\title{
SOME EXAMPLES OF VANISHING YAMABE INVARIANT AND MINIMAL VOLUME, AND COLLAPSING OF INEQUIVALENT SMOOTHINGS AND PL-STRUCTURES
}

\author{
RAFAEL TORRES
}

\begin{abstract}
In this short note, exploits of constructions of $\mathcal{F}$-structures coupled with technology developed by Cheeger-Gromov and Paternain-Petean are seen to yield a procedure to compute minimal entropy, minimal volume, Yamabe invariant and to study collapsing with bounded sectional curvature on inequivalent smooth structures and inequivalent PL-structures within a fixed homeomorphism class. We compute these fundamental Riemannian invariants for every high-dimensional smooth manifold on the homeomorphism class of any smooth manifold that admits a Riemannian metric of zero sectional curvature. This includes all exotic and all fake tori of dimension greater than four. We observe that the minimal volume is not an invariant of the smooth structures, yet the Yamabe invariant does discern the standard smooth structure from all the others. We also observe that the fundamental group places no restriction on the vanishing of the minimal volume and collapse with bounded sectional curvature for high-dimensional manifolds.
\end{abstract}

\section{INTRODUCTION AND MAIN RESULTS}

Within a fixed homeomorphism class, we refer as inequivalent smoothings to different smooth structures up to diffeomorphism and as inequivalent PL-structures to different PL-structures up to PL-homeomorphism. The first examples of inequivalent smoothings were provided by Milnor [M56] on the homeomorphism class of the 7-sphere. A classical success of surgery theory established the existence of both inequivalent smoothings and inequivalent PL-structures on high-dimensional manifolds W69. A manifold homeomorphic to an n-sphere is known as a homotopy sphere. Hsiang-Wall [HW69] have shown that a closed n-manifold that is homotopy equivalent to the $\mathrm{n}$-torus is homeomorphic to it provided $n \geq 5$. Such a manifold is known as a homotopy n-torus. Farrell-Jones [FJ98 have proven the Borel conjecture for closed Riemannian manifolds with non-negative sectional curvature. Motivated by their result, we call a homotopy Euclidean space n-form a closed n-manifold that is homotopy equivalent to $\mathbb{E}^{n} / \Gamma$.

It is known that inequivalent smoothings need not share certain basic geometric properties with the standard smooth structure. Hitchin has shown in [H74 that certain homotopy spheres do not admit a Riemannian metric of positive scalar curvature, while the round metric on the standard $S^{n}$ has positive sectional curvature. When it comes to computations of fundamental invariants in Riemannian geometry, Euclidean geometry canonically serves as a basic model; see Section 2.2 for definitions of the invariants studied in this paper. For example, among the many interesting geometric traits of an n-dimensional compact Euclidean space form $\mathbb{E}^{n} / \Gamma$ 
are the existence of Riemannian metrics of zero sectional curvature, hence the vanishing and realization of its Yamabe invariant. Gromov's minimal volume is zero for $\mathbb{E}^{n} / \Gamma$, and so is its minimal entropy. Moreover, Riemannian manifolds with zero sectional curvature make prototypes to study collapsing since they observe that collapse by rescalling preserves bounded sectional curvature. Although these invariants play a fundamental role in Riemannian geometry, their computations are as challenging as they are scarce.

We observe in this note that exploits of $\mathcal{F}$-structures and results of Gromov G82, Cheeger-Gromov [CG86, and Paternain-Petean [PP1], among others, yield a procedure to calculate the aforementioned invariants on inequivalent smoothings and PL-structures for manifolds of dimension at least five. This procedure enlarges considerably the set of examples for which the values of the invariants are known. We show that the minimal volume does not distinguish different smooth nor PLstructures on a homotopy Euclidean space form, while the Yamabe invariant does discern the standard smooth structure. The precise statement is as follows.

Theorem A. Every homotopy Euclidean space $n$-form $M$ with $n \geq 5$ admits a polarized $\mathcal{F}$-structure. Consequently,

$$
\operatorname{Min} \operatorname{Vol}(M)=0,
$$

and $M$ collapses with bounded sectional curvature.

The Yamabe invariant satisfies

$$
\mathcal{Y}(M)=0,
$$

and it is realized, i.e., there exists a scalar-flat Riemannian metric on $M$ if and only if $M$ is diffeomorphic or PL-homeomorphic to the Euclidean space form $\mathbb{E} / \Gamma$.

Theorem A can be compared to results in B98 and BT12 (see Remark1 for a suggested extension). Bessières has shown that the minimal volume is sensitive to inequivalent smoothings on homeomorphism types of hyperbolic manifolds B98. Inequivalent smoothings and PL-structures on the n-torus for were shown to exist by Casson, Wall, and Hsiang-Shaneson HS69, HW69, provided $n \geq 5$. They are respectively known as exotic and fake tori, and they are all addressed by Theorem A. The torus $T^{n}=S^{1} \times \cdots \times S^{1}$ is called the standard n-torus for $n \in \mathbb{N}$.

Corollary B. Every homotopy $n$-torus $M$ with $n \geq 5$ admits a polarized $\mathcal{F}$ structure. Consequently,

$$
\operatorname{Min} \operatorname{Vol}(M)=0,
$$

and $M$ collapses with bounded sectional curvature.

The Yamabe invariant satisfies

$$
\mathcal{Y}(M)=0,
$$

and it is realized, i.e., there exists a scalar-flat Riemannian metric on $M$ if and only if $M$ is diffeomorphic or PL-homeomorphic to the $n$-torus $S^{1} \times \cdots \times S^{1}$.

Baues-Tuschmann have shown that the vanishing of the more restrictive invariant D-MinVol does discern the standard smooth structure on the n-torus from certain inequivalent smoothings (see Section 2.2 for the definition of the invariant). Corollary $\mathrm{B}$ says this is not the case for MinVol of any homotopy n-torus. Moreover, we observe the following corollary of Baues-Tuschmann's work. 
Proposition C. Within the homeomorphism type of the $n$-torus for $n \geq 5$ there are smoothings and PL-structures for which $\mathrm{D}-\mathrm{MinVol}$ decreases under finite coverings.

On the other hand, for every choice of smoothing or PL-structure, MinVol is invariant under finite coverings.

Corollary B answers a question of Baues-Tuschmann BT12; see Remark 2, Theorem $\mathrm{A}$ and the results in this note can be generalized to many more inequivalent smoothings and PL-structures on a broader range of homeomorphism types (see Corollary 1, Theorem 4, and Remark 1).

We conclude the note by showing that the fundamental group places no restriction on the vanishing of the minimal volume and collapse with bounded sectional curvature for manifolds of dimension greater than four.

Theorem D. Let $G$ be a finitely presented group and suppose $n \geq 5$. There exists a closed smooth $n$-manifold $M(G)$ with fundamental group $\pi_{1}(M(G)) \cong G$ that admits a polarized $\mathcal{T}$-structure. Consequently,

$$
\operatorname{MinVol}(M(G))=0,
$$

and $X(G)$ collapses with bounded sectional curvature.

Cheeger-Rong CR96, Corollary 0.3] have shown that in some sense most manifolds with bounded sectional curvature have minimal volume zero.

The note is structured as follows. The definitions, machinery and main results that we build upon are recollected in Section 2. Section 2.1 contains the definition of $\mathcal{F}$-structures, the principal tool of this paper, as well as several useful examples and constructions that include all $\mathcal{F}$-structures of Theorem $\mathrm{A}$ its corollary, and Theorem D, The definitions of the Riemannian invariants considered in this paper and the results we build upon are collected in Section 2.2 The vanishing and nonrealization of the Yamabe invariant follows from the main results of Section 2.2 and Section 2.3. Existence of $\mathcal{F}$-structures on exotic spheres and homotopy real projective spaces is considered in Section 2.4. Homotopy Euclidean space forms are studied in Section 2.5. It contains a description of the results of surgery theory that we employ. The proofs of Theorem $\mathrm{A}$. Corollary B Proposition $\mathrm{C}$ and Theorem $\mathrm{D}$ are given in Section 3.1 .

\section{Definitions AND BACKGROUnd RESUlts}

We collect in this section the definitions and main results used in this manuscript in order to make it as self-contained as possible.

2.1. $\mathcal{F}$-structures and constructions. The definition of an $\mathcal{F}$-structure below was given in [PP09, Section 2] and it is equivalent to the original one introduced by Cheeger-Gromov [G82, CG86].

Definition 1. An $\mathcal{F}$-structure on a smooth closed manifold $M$ is given by

(1) a finite open cover $\left\{U_{1}, \ldots, U_{N}\right\}$ of $M$;

(2) a finite Galois covering $\pi_{i}: \widetilde{U}_{i} \rightarrow U_{i}$ with $\Gamma_{i}$ a group of deck transformations for $1 \leq i \leq N$;

(3) a smooth effective torus action with finite kernel of a $k_{i}$-dimensional torus

$$
\phi_{i}: T^{k_{i}} \rightarrow \operatorname{Diff}\left(\widetilde{U_{i}}\right)
$$


for $1 \leq i \leq N$;

(4) a representation $\Phi_{i}: \Gamma_{i} \rightarrow \operatorname{Aut}\left(T^{k_{i}}\right)$ such that

$$
\gamma\left(\phi_{i}(t)(x)\right)=\phi_{i}\left(\Phi_{i}(\gamma)(t)\right)(\gamma x)
$$

for all $\gamma \in \Gamma_{i}, t \in T^{k_{i}}$, and $x \in \widetilde{U}_{i}$;

(5) for any subcollection $\left\{U_{i_{1}}, \ldots, U_{i_{l}}\right\}$ that satisfies

$$
U_{i_{1} \cdots i_{l}}:=U_{i_{1}} \cap \cdots \cap U_{i_{l}} \neq \emptyset,
$$

the following compatibility condition holds: let $\widetilde{U}_{i_{1} \cdots i_{l}}$ be the set of all points $\left(x_{i_{1}}, \ldots, x_{i_{l}}\right) \in \widetilde{U}_{i_{1}} \times \cdots \times \widetilde{U}_{i_{l}}$ such that $\pi_{i_{1}}\left(x_{i_{1}}\right)=\cdots=\pi_{i_{l}}\left(x_{i_{l}}\right)$. The set $\widetilde{U}_{i_{1} \cdots i_{l}}$ covers $\pi^{-1}\left(U_{i_{1} \cdots i_{l}}\right) \subset \widetilde{U}_{i_{1} \cdots i_{l}}$ for all $1 \leq j \leq l$. It is required that $\phi_{i_{j}}$ leaves $\pi_{i_{j}}^{-1}\left(U_{i_{1} \cdots i_{l}}\right)$ invariant, and it lifts to an action on $\widetilde{U}_{i_{1} \cdots i_{l}}$ such that all lifted actions commute.

(6) An $\mathcal{F}$-structure is called a $\mathcal{T}$-structure if the Galois coverings $\pi_{i}: \widetilde{U}_{i} \rightarrow U_{i}$ in Item (1) can be taken to be trivial for every $i$.

(7) The $\mathcal{F}$-structure is said to be polarized if the torus action $\phi_{i}$ of Item (3) is fixed-point free $\forall$ open set $U_{i}$.

(8) The $\mathcal{F}$-structure is pure if the dimension of the orbit of the torus action $\phi_{i}$ of Item (3) is constant for every point $x \in \widetilde{U}_{i}$ and every $i$.

A non-trivial circle action yields a $\mathcal{T}$-structure. We now discuss two warm-up examples.

Example 1. Non-pure $\mathcal{T}$-structure on $S^{n}$ and non-pure $\mathcal{F}$-structure on $\mathbb{R} P^{n}$ that are polarized if $n$ is odd. Write the $\mathrm{n}$-sphere as

$$
S^{n}=\partial D^{n+1}=\partial D^{2} \times D^{n-1}=\left(S^{1} \times D^{n-1}\right) \cup_{\mathrm{id}}\left(D^{2} \times S^{n-2}\right)
$$

where the pieces on the right hand side of (6) are identified along the common boundary $S^{1} \times S^{n-2}$ by the identity map. We use this decomposition of $S^{n}$ to construct a $\mathcal{T}$-structure as follows. Define as the covers of Items (1) and (2) of Definition 1

$$
\widetilde{U}_{1}=U_{1}:=S^{1} \times D^{n-1}
$$

and

$$
\widetilde{U}_{2}=U_{2}:=D^{2} \times S^{n-2} .
$$

These choices imply that Item (4) does not need to be checked. Regarding Item (3), we have the following. The circle acts by rotations on the $S^{1}$ factor of $U_{1}$, and we label it

$$
\phi_{1}: S^{1} \rightarrow \operatorname{Diff}\left(U_{1}\right) .
$$

This is a free action. Take a circle action on the $S^{n-2}$-factor of $U_{2}$ and label it

$$
\phi_{2}: S^{1} \rightarrow \operatorname{Diff}\left(U_{2}\right) .
$$

If $n$ is odd, then $\phi_{2}$ can be chosen to be fixed point free, i.e., as the Hopf action. There is a $T^{2}$-action on the common boundary induced by $\phi_{1}$ and $\phi_{2}$, hence Item (5) is verified. Our choices (7) and (8) comply with Item (6) in the definition. We have thus constructed a non-pure $\mathcal{T}$-structure on the $n$-sphere that is polarized in the odd-dimensional case. 
The real projective n-space is the orbit space $S^{n} / T$ of the antipodal involution $T: S^{n} \rightarrow S^{n}$ that identifies $x \mapsto-x$ for $x \in S^{n}$. The ecomposition (6) yields

$$
\mathbb{R} P^{n}=\left(D^{n-1} \widetilde{\times} S^{1}\right) \cup \text { id }\left(D^{2} \widetilde{\times} \mathbb{R} P^{n-2}\right)
$$

We point out the following abuse of notation that will carry for the remaining discussion of this example. If $n$ is even, the symbol $D^{n-1} \widetilde{\times} S^{1}$ denotes the nonorientable (n - 1)-disk bundle over the circle, and $D^{2} \widetilde{\times} \mathbb{R} P^{n-2}$ is the twisted nonorientable 2disk bundle over the real projective (n - 2)-space. In the orientable case, i.e., when $n$ is odd, the symbol $D^{n-1} \widetilde{\times} S^{1}$ simply denotes the trivial bundle $D^{n-1} \times S^{1}$, while $D^{2} \widetilde{\times} \mathbb{R} P^{n-2}$ is the twisted orientable bundle. A construction of an $\mathcal{F}$-structure on $\mathbb{R} P^{n}$ suggested by these decompositions is as follows. Define

$$
U_{1}:=D^{n-1} \widetilde{\times} S^{1}=\left(S^{1} \times D^{n-1}\right) / T
$$

and

$$
U_{2}:=D^{2} \widetilde{\times} \mathbb{R} P^{n-2}=\left(D^{2} \times S^{n-2}\right) / T .
$$

Their covers $\widetilde{U}_{1}$ and $\widetilde{U}_{2}$ are respectively given in (7) and (8) with $\Gamma_{i}=\mathbb{Z} / 2$ as in Items (1) and (2) in Definition 1. We choose the same circle actions $\phi_{1}$ and $\phi_{2}$ as in (9) and (10), and Items (3) and (5) hold. The Deck transformation given by the involution $T$ is the antipodal map on each piece. We use it to definite the homeomorphism $\Phi_{i}: \mathbb{Z} / 2 \rightarrow \operatorname{Aut}\left(S^{1}\right) \cong \mathbb{Z} / 2$ as $t \mapsto-t$ for $t \in \Gamma_{i}$ as in Item (4). Moreover, both $\phi_{1}$ and $\phi_{2}$ commute with $T$. If $n$ is odd, we can choose $\widetilde{U}_{1}=U_{1}$ and $\phi_{2}$ is free. We have then constructed a non-pure $\mathcal{F}$-structure on the real projective $\mathrm{n}$-space that is polarized if its dimension is odd.

Example 2. Pseudofree circle actions give rise to polarized $\mathcal{T}$-structures. A circle action $\phi: S^{1} \rightarrow \operatorname{Diff}(M)$ on a smooth manifold $M$ is said to be pseudofree if it is not free, every orbit is one-dimensional, and if the isotropy group of $\phi$ is the identity except for isolated exceptional orbits, where it is a finite cyclic group [MY68. Since all isotropy groups of such an action are discrete, the action is locally-free.

Our constructions of $\mathcal{F}$-structures in this section continue with a proof of the first claim of Theorem $D$

Proposition 1. Let $G$ be a finitely presented group and suppose $n \geq 5$. There exists a closed smooth n-manifold $X(G)$ with fundamental group $\pi_{1}(X(G)) \cong G$ that admits a polarized $\mathcal{T}$-structure.

A classical result of Dehn states the existence of a smooth closed orientable $n$ manifold $X(G)$ whose fundamental group is isomorphic to any finitely presented group $G$ for every integer $n \geq 4$ (cf. D12]). It trivially follows that for any $k \geq 7$ and $k \neq 8$ there is a $k$-manifold $M(G)$ with arbitrary finitely presented fundamental group that admits a polarized $\mathcal{T}$-structure by taking

$$
M(G):=X(G) \times \underbrace{S^{3} \times \cdots \times S^{3}}_{r} \times \underbrace{S^{2} \times \cdots \times S^{2}}_{s}
$$

with $r \in \mathbb{N}$ and $s \in \mathbb{Z}_{\geq 0}$ and equip it with a free circle action on a 3 -sphere factor. In order to prove Proposition 1, we need to construct such a manifold in dimensions five, six, and eight. We proceed to do so using cut-and-paste constructions in every dimension, thus yielding a different manifold and $\mathcal{T}$-structure to the one described in (14). We give two constructions of $\mathcal{T}$-structures arising from surgery along loops and from surgery along embedded tori. Each piece in the assembly is equipped with 
a torus action and all these actions commute along the common boundary where the pieces are glued together as it was sampled in Example 1.

Proof. We first discuss the construction on an odd-dimensional manifold with arbitrary finitely presented fundamental group using surgery along loops. Let $X(G)$ be a closed smooth $(n-1)$-manifold of even dimension and $\pi_{1}(X(G)) \cong G[\mathrm{D} 12$ and take the odd-dimensional $n$-manifold $X(G) \times S^{1}$ with fundamental group $G \times \mathbb{Z}$ for $n \geq 5$. A tubular neighborhood of the circle factor $\{x\} \times S^{1} \subset X(G) \times S^{1}$ is diffeomorphic to $D^{n-1} \times S^{1}$. We carve out this tubular neighborhood and construct the closed smooth $n$-manifold

$$
M(G):=\left(X(G) \times S^{1} \backslash D^{n-1} \times S^{1}\right) \cup_{\text {id }}\left(S^{n-2} \times D^{2}\right),
$$

where the pieces are identified along their common $S^{n-2} \times S^{1}$ boundaries using the identity map. The Seifert-van Kampen theorem implies that the fundamental group of $M(G)$ is isomorphic to $G$. The isotopy class of the loop $\{x\} \times S^{1} \subset X(G) \times S^{1}$ is the representative for the homotopy class of the generator of the infinite cyclic factor in the group $\pi_{1}\left(X(G) \times S^{1}\right)=G \times \mathbb{Z} t$. The induced identification from (15) sets $t=0$ in $\pi_{1}(M(G))$ and this group is isomorphic to $G$.

The choice of coverings and circle actions of Definition 1 are the following. We pick the first piece of $M(G)$ as the complement of the aforementioned tubular neighborhood inside $X(G) \times S^{1}$, and set

$$
\widetilde{U}_{1}=U_{1}:=X(G) \times S^{1} \backslash\left(D^{n-1} \times S^{1}\right) .
$$

It has an induced circle action $\phi_{1}: S^{1} \rightarrow \operatorname{Diff}\left(U_{1}\right)$ from the free circle action on the $S^{1}$ factor of $X(G) \times S^{1}$. The second piece is chosen as

$$
\widetilde{U}_{2}=U_{2}:=S^{n-2} \times D^{2} .
$$

We take a free circle action $\phi_{2}: S^{1} \rightarrow \operatorname{Diff}\left(S^{n-2} \times D^{2}\right)$ induced from a free circle action on the sphere $S^{n-2} \times\{d\} \subset S^{n-2} \times D^{2}$ for $d \in D^{2}$, which is odd-dimensional. This is the only part of the proof that requires $n$ to be odd. These considerations verify Items (1), (2), and (3) of Definition 1. Notice that since the Galois covering of Item (2) is trivial, Item (4) holds automatically. Item (5) holds due to our choices of circle actions. There is a $T^{2}$-action $\left(\phi_{2}, \phi_{1}\right)$ on the $S^{n-2} \times S^{1}$ boundary of each piece; each circle action acts on a factor. Our choice of covering satisfies condition of Item (6). Since both actions $\phi_{1}$ and $\phi_{2}$ are fixed-point free, the condition in Item (7) holds and we have constructed a polarized $\mathcal{T}$-structure on an n-dimensional manifold $M(G)$ with finitely presented fundamental group $G$ for every odd dimension $n \geq 5$.

We finish the proof of Proposition 1 describing the construction of $\mathcal{T}$-structures using surgeries along embedded tori. This cut-and-paste technique not only settles the even-dimensions case, but it also provides a proof of the proposition in all dimensions. We describe the construction for the 6-dimensional case for the sake of a clear exposition, and we proceed to do so. Gompf [GS99, Theorem 10.2.10] constructed a closed smooth 4-manifold $Y(G)$ with $\pi_{1}(Y(G)) \cong G$ and that contains two disjoint 2-tori $\left\{T_{1}, T_{2}\right\}$ of self-intersection zero. Their tubular neighborhoods $\nu\left(T_{i}\right) i=1,2$ are diffeomorphic to $D^{2} \times T^{2}$ and since each torus intersects a 2sphere inside $Y(G)$ GS99, Theorem 10.2.10], we have $\pi_{1}\left(Y(G) \backslash\left(\nu\left(T_{1}\right) \cup \nu\left(T_{2}\right)\right)\right) \cong$ $\pi_{1}(Y(G))$. Consider the 6 -dimensional manifold $Y(G) \times S^{1} \times S^{1}$ with fundamental group $G \times \mathbb{Z} \times \mathbb{Z}$. 
The manifold $M(G)$ is obtained by carving out two disjoint embedded 4-tori

$$
T_{i}^{4}:=T_{i}^{2} \times S^{1} \times S^{1}
$$

inside $Y(G) \times S^{1} \times S^{1}$ and gluing them back in with an specified choice of diffeomorphism of the common boundary. A tubular neighborhood $\nu\left(T_{i}^{4}\right)$ is diffeomorphic to

$$
D^{2} \times T^{2} \times S^{1} \times S^{1}
$$

since $T_{i}^{4}$ has zero self-intersection for $i=1,2$. We build our manifold as

$$
M(G):=\left(Y(G) \times S^{1} \times S^{1} \backslash \nu\left(T_{1}^{4}\right) \cup \nu\left(T_{2}^{4}\right)\right) \cup\left(\bigsqcup_{i=1}^{2}\left(D_{i}^{2} \times T^{2} \times S^{1} \times S^{1}\right)\right)
$$

with the following choices of diffeomorphisms

$$
\varphi_{i}: \partial D_{i}^{2} \times T^{2} \times S^{1} \times S^{1} \longrightarrow \partial D^{2} \times T^{2} \times S^{1} \times S^{1}
$$

of the boundary. Notice that the subindex associated to the 2-disk as $D_{i}^{2}$ for $i=1,2$ discerns the carved torus $T_{i}^{4}$ that is being glued back in. We choose the diffeomorphism $\varphi_{1}: \partial D_{1}^{2} \times T^{2} \times S^{1} \times S^{1} \rightarrow \partial D^{2} \times T^{2} \times S^{1} \times S^{1}$ that identifies $\partial D^{2}$ with the circle factor $\left\{\theta_{0}\right\} \times\{t\} \times S^{1} \times\left\{\theta_{2}\right\}$ for $\theta_{j} \in S^{1}$ and $t \in T^{2}$. Analogously, we use the diffeomorphism $\varphi_{2}: \partial D_{2}^{2} \times T^{2} \times S^{1} \times S^{1} \rightarrow \partial D^{2} \times T^{2} \times S^{1} \times S^{1}$ that identifies $\partial D^{2}$ with the circle factor $\left\{\theta_{0}\right\} \times\{t\} \times\left\{\theta_{1}\right\} \times S^{1}$. These operations are high-dimensional generalization of the multiplicity zero logarithmic transformations of 4-manifolds GS99.

We use the Seifert-van Kampen theorem to conclude that the fundamental group of $M(G)$ is isomorphic to $G$ as follows. Both meridians of the 4-tori that we removed are nullhomotopic in the complement and thus we have an isomorphism $\pi_{1}\left(Y(G) \times S^{1} \times S^{1} \backslash\left(\nu\left(T_{1}^{4}\right) \cup \nu\left(T_{2}^{4}\right)\right) \cong \pi_{1}(Y(G))\right) \times \mathbb{Z} t \times \mathbb{Z} s$. Each isotopy class of the two loops $\{y\} \times S^{1} \times\left\{\theta_{2}\right\} \subset Y(G) \times S^{1} \times S^{1}$ and $\{y\} \times\left\{\theta_{1}\right\} \times S^{1} \subset Y(G) \times S^{1} \times S^{1}$ is the representative for the homotopy class of the generator $t$ and sof the infinite cyclic factor in the group $\pi_{1}\left(Y(G) \times S^{1} \times S^{1}\right)=G \times \mathbb{Z} t \times \mathbb{Z} s$. The induced identifications from the specified diffeomorphisms (21) set $t=0$ and $s=0$ in the group $\pi_{1}(M(G)$ ), which implies that it is isomorphic to $G$.

We construct the polarized $\mathcal{T}$-structure as follows. The open sets of the coverings of Items (1) and (2) in Definition[1are defined following the deconstruction of $M(G)$ in (20) as

$$
\begin{gathered}
\widetilde{U}_{1}=U_{1}:=Y(G) \times S^{1} \times S^{1} \backslash\left(\left(D^{2} \times T_{1} \times S^{1} \times S^{1}\right) \cup\left(D^{2} \times T_{2} \times S^{1} \times S^{1}\right)\right), \\
\widetilde{U}_{2}=U_{2}:=D^{2} \times T_{1} \times S^{1} \times S^{1}=D^{2} \times T^{2} \times S^{1} \times S^{1}
\end{gathered}
$$

and

$$
\widetilde{U}_{3}=U_{3}:=D^{2} \times T_{2} \times S^{1} \times S^{1}=D^{2} \times T^{2} \times S^{1} \times S^{1} .
$$

We choose a free circle action on each $U_{i}$ as follows. On $U_{1}$, we pick the free circle action $\phi: S^{1} \rightarrow \operatorname{Diff}\left(U_{1}\right)$ induced by the free action of the circle factor $\{y\} \times S^{1} \times\left\{\theta_{2}\right\} \subset Y(G) \times S^{1} \times S^{1}$ for $y \in Y(G)$ and $\theta_{2} \in S^{1}$ acting on itself. We choose $\phi_{2}: S^{1} \rightarrow \operatorname{Diff}\left(D^{2} \times T^{2} \times S^{1} \times S^{1}\right)$ to be a circle action on the first circle of the 2-torus factor of $\{d\} \times T^{2} \times\left\{\theta_{1}\right\} \times\left\{\theta_{2}\right\}=\{d\} \times\left(S^{1} \times S^{1}\right) \times\left\{\theta_{1}\right\} \times\left\{\theta_{2}\right\} \subset U_{2}$ for $d \in D^{2}$ and $\theta_{j} \in S^{1}$. Similarly, $\phi_{3}: S^{1} \rightarrow \operatorname{Diff}\left(D^{2} \times T^{2} \times S^{1} \times S^{1}\right)$ is chosen to be the circle action of the second circle of the $T^{2}$ factor in $U_{3}$ acting on itself. 
This verifies Item (3) in Definition 1, and Item (4) does not need to be considered in this case since $\Gamma_{i}=\{1\}$.

The actions $\phi_{i}$ for $i=1,2,3$ paste together to a $T^{3}$-action on the boundary $S^{1} \times T^{2} \times S^{1} \times S^{1}$, hence the condition of Item (5) is met and so is the on of Item (6). Since all three actions are free, Item (7) is satisfied and we have constructed a polarized $\mathcal{T}$-structure on a closed smooth 6 -manifold $M(G)$ with fundamental group $G$. As we have mentioned, the argument presented is readily extended to dimension greater or equal to five. This concludes the proof of the proposition.

Soma [S81] showed that existence of polarized $\mathcal{T}$-structures on 3-manifolds is closed under connected sums, and Gromov [G82, Appendix 2] (cf. CG86, Example A.1]) remarked that the result holds in all odd dimensions. With broader generality, Paternain-Petean have shown that the existence of $\mathcal{T}$-structures is closed under connected sums of closed manifolds of dimensions greater than two PP03, Theorem $5.9]$.

Proposition 2. Soma, Gromov. Let $X$ and $Y$ be closed smooth $(2 k+1)$-manifolds with $k \in \mathbb{N}$ that admit a polarized $\mathcal{F}$-structure. Assume that the open cover of each $\mathcal{F}$-structure contains at least one open set with trivial Galois covering. Their connected sum $X \# Y$ admits a polarized $\mathcal{F}$-structure.

The following proof of Proposition 2 is due to to Paternain-Petean [PP03, Proof of Theorem 5.9]; it is a word by word repetition of their argument modulo a small tweak to justify the addition of the adjective 'polarized'. The proof is included for the sake of completeness.

Proof. Paternain-Petean's construction of the polarized $\mathcal{F}$-structure on $X \# Y$ is as follows. The connected sum $X \# Y$ is deconstructed into a union of three pieces. Each piece is equipped with a polarized $\mathcal{F}$-structure such that the $\mathcal{T}$-structures induced on adjacent boundary components are compatible with each other (see Definition (1). The three polarized $\mathcal{F}$-structures then glue together to a (global) polarized $\mathcal{F}$-structure on $X \# Y$.

Begin by finding embedded solid tori

$$
S^{1} \times D_{x} \hookrightarrow X \text { and } S^{1} \times D_{y} \hookrightarrow Y,
$$

where $D_{x}$ and $D_{y}$ are small $2 k$-balls centered at points $x \in X$ and $y \in Y$ respectively. We describe this step of the procedure just for $X$ given that it is the very same for $Y$. If needed, modify the $\mathcal{F}$-structure on $X$ and take a point $x \in X$ contained in a single open set $U_{j}$ (for a fixed $j$ ) of the polarized $\mathcal{F}$-structure that has trivial Galois covering as in Item (2) of Definition 1. Without loss of generality, we can assume that the corresponding torus $T^{k_{j}}$ acting on $U_{j}$ is a circle, and that the point $x$ is contained in a regular orbit. The small $2 k$-ball $D_{x}$ centered at $x$ is taken to be transverse to this circle action. The embedded solid torus $S^{1} \times D_{x}$ is obtained as the union of orbits through $D_{x}$. At this point of the proof we have located embedded solid tori inside $X$ and $Y$.

The connected sum is to be performed inside these tori. We use the existence of a diffeomorphism between $S^{1} \times D_{x} \# S^{1} \times D_{y}$ and $\left(S^{1} \times D_{x} \backslash S^{2 k-1} \times D^{2}\right)$, where the boundary component coming from the $S^{2 k-1} \times D^{2}$ piece that was carved out is identified with the boundary $\partial\left(S^{1} \times D_{y}\right)=S^{1} \times S^{2 k-1}$. To see the decomposition, 
split the $2 k$-disk around $x$ as

$$
D_{x}=D_{\epsilon_{1}} \cup\left(S^{2 k-1} \times\left[\epsilon_{1}, \epsilon_{2}\right]\right),
$$

where $D_{\epsilon_{1}}$ for small constants $\epsilon_{1}, \epsilon_{2}$. The 2-disk factor $\{p t\} \times D^{2} \subset S^{2 k-1} \times D^{2}$ is a small 2-disk around a point contained in the middle of $S^{2 k-1} \times\left[\epsilon_{1}, \epsilon_{2}\right]$ and transverse to $\{p t\} \times S^{2 k-1} \subset S^{1} \times S^{2 k-1} \times\left[\epsilon_{1}, \epsilon_{2}\right]$.

The manifold $X \# Y$ is constructed as the union of the pieces

$$
\begin{gathered}
\left(X \backslash S^{1} \times D_{x}\right) \cup S^{1} \times D_{\epsilon} \\
S^{1} \times S^{2 k-1} \times\left[\epsilon_{1}, \epsilon_{2}\right] \backslash\left(S^{2 k-1} \times D^{2}\right)
\end{gathered}
$$

and

$$
Y \backslash S^{1} \times D_{y} .
$$

The choices of $\mathcal{F}$-structures are the following. Equip pieces (26) and (28) with the initial $\mathcal{F}$-structure, which is polarized by hypothesis. Take a free circle action on the $S^{2 k-1}$ factor of $S^{1} \times S^{2 k-1} \times\left[\epsilon_{1}, \epsilon_{2}\right] \backslash\left(S^{2 k-1} \times D^{2}\right)$. This is the point of the proof where the hypothesis on the dimension of the manifolds to be $2 k+1 \geq 3$ is used. This equips piece (27) with a polarized $\mathcal{T}$-structure. To see that these structures are compatible on the boundary components, we argue as follows. The action induced on each boundary component of pieces (26), (27), and (28) by our choices of $\mathcal{F}$-structures pastes together to the canonical action on the circle factor into a $T^{2}$-action. Hence, the conditions of Definition 1 are satisfied, and the connected sum $X \# Y$ has a polarized $\mathcal{F}$-structure.

We sample in the following corollary the utility of the previous proposition to equip inequivalent smoothings with a polarized $\mathcal{F}$-structure.

Corollary 1. - There are 28 inequivalent smoothings of $\mathbb{R} P^{7}$ that admit a polarized $\mathcal{T}$-structure.

- Any homeomorphism class that is realized by a closed flat 7-manifold has 28 inequivalent smoothings that admit a polarized $\mathcal{F}$-structure.

To prove Corollary 1, we use well-known argument to construct inequivalent smoothings on high-dimensional manifolds.

Proof. There is a free circle action on the real projective 7 -space, which yields a polarized $\mathcal{T}$-structure. Consider the connected sum $\mathbb{R} P^{7} \# \Sigma^{7}$, which is homeomorphic to the projective 7-space. Montgomery-Yang MY68 have shown that every homotopy 7-sphere admits a pseudo free circle action. Example2 and Proposition 2 imply that any such connected sum admits a polarized $\mathcal{T}$-structure. We can choose homotopy 7-spheres $\Sigma_{i}^{7}$ and $\Sigma_{j}^{7}$ such that looking at the universal covers $S^{7} \# \Sigma_{i} \# \Sigma_{i}$ and $S^{7} \# \Sigma_{j} \# \Sigma_{j}$ of the two homeomorphic manifolds $\mathbb{R} P^{7} \# \Sigma_{i}^{7}$ and $\mathbb{R} P^{7} \# \Sigma_{j}^{7}$, we conclude that they are diffeomorphic if and only if $i=j$. The corollary now follows since there are 28 smoothings on $S^{7}$.

The proof of the second claim is similar and we present it without assumptions on the dimension. Let $\Sigma^{n}$ be a homotopy n-sphere and set $X=\mathbb{E}^{n} / \Gamma$. CheegerGromov have shown that $X$ admits a polarized $\mathcal{F}$-structure [CG86]. The connected sum

$$
X \# \Sigma^{n}
$$


is homeomorphic to the Euclidean space form $\mathbb{E}^{n} / \Gamma$ but it need not be diffeomorphic to it (cf. W99, Chapter 15]).

The next proposition generalizes CG86, Examples 0.3 and 1.3]. It will be used in Section 2.5 and in the proof of Theorem A.

Proposition 3. Let $\widetilde{M}$ and $M$ be closed smooth n-manifolds and let

$$
\widetilde{M} \stackrel{\pi}{\longrightarrow} M
$$

be a finite covering. Suppose $\widetilde{M}$ admits a free torus action

$$
\widetilde{\phi}: T^{k} \rightarrow \operatorname{Diff}(\widetilde{M}) .
$$

There is a polarized $\mathcal{F}$-structure on $M$.

Proof. The free torus action $\widetilde{\phi}$ yields a pure polarized $\mathcal{T}$-structure on $\widetilde{M}$ with elements of the covering $\left\{V_{1}, \ldots, V_{N}\right\}$ given by embedded submanifolds that are diffeomorphic to the torus orbits. We use this structure to construct a polarized $\mathcal{F}$ structure on $M$. Regarding Item (1) of Definition 1, choose a covering $\left\{U_{1}, \ldots, U_{N}\right\}$ for $M$ of open sets whose intersection are either empty or path-connected and such that elements of $\left\{\pi^{-1}\left(U_{i}\right)\right\}$ are diffeomorphic to $U_{i}$ for every $i \in\{1, \ldots, N\}$. Pick a Riemannian metric $g$ on $M$ and let $\tilde{g}$ be the metric on $\widetilde{M}$ that makes $\pi$ a Riemannian submersion $(\widetilde{M}, \tilde{g}) \rightarrow(M, g)$. Fix points $x \in M$ and $y \in \pi^{-1}(x) \subset \widetilde{M}$, and take an $x_{i} \in U_{i}$ for every $i$. Let $\alpha_{i}:[0,1] \rightarrow M$ be a geodesic with initial and end points $\alpha_{i}(0)=x$ and $\alpha_{i}(1)=x_{i}$. There is a geodesic $\widetilde{\alpha}_{i}:[0,1] \rightarrow \widetilde{M}$ with $\widetilde{\alpha}_{i}(0)=y$ and $\widetilde{\alpha}_{i}(1)=\widetilde{x}_{i} \in \pi^{-1}\left(x_{i}\right)$ and $\widetilde{x}_{i} \in \widetilde{U}_{m}$ for some open subset $\widetilde{U}_{i} \subset \widetilde{M}$. We now use the $T^{k}$-torus action of (31) to define an action $\phi_{i}: T^{k_{i}} \rightarrow \operatorname{Diff}\left(\widetilde{U}_{i}\right)$ with trivial isotropy group as in Items (3) and (7) of Definition 1. Notice that the hypothesis on $\widetilde{\phi}$ can be relaxed as follows. For any action $T^{k} \rightarrow \operatorname{Diff}(\widetilde{M})$ whose isotropy group has codimension greater than one, we immediately obtain a free circle action for a given $S^{1} \subset T_{\sim}^{k}$. We can define a circle action as the aforementioned restriction of the torus action $\widetilde{\phi}: T^{k} \rightarrow \operatorname{Diff}\left(V_{j}\right)$ to $\widetilde{U}_{i} \subset \widetilde{M}$ for every $1 \leq i \leq N$. If the intersections $U_{i} \cap U_{j}$ and $\widetilde{U}_{i} \cap \widetilde{U}_{j}$ are both nonempty, we have a canonical covering. If the intersection $U_{i} \cap U_{j}$ is empty, yet $\widetilde{U}_{i} \cap \widetilde{U}_{j} \neq \emptyset$, then there is a Deck transformation $g: \widetilde{M} \rightarrow \widetilde{M}$ such that $g \widetilde{U}_{i} \cap \widetilde{U}_{j} \neq \emptyset$. The corresponding group of Deck transformations on the open set is finite by assumption and $\operatorname{Aut}\left(S^{1}\right) \cong \mathbb{Z} / 2$.. The commutativity condition in Item (4), and invariance of Item (5) of Definition 1 are immediately satisfied. Hence, we have constructed a polarized $\mathcal{F}$-structure on $M$.

2.2. Collapsing, minimal volumes and entropy, and Yamabe invariant. A smooth manifold $M$ collapses with bounded sectional/Ricci/scalar curvature if and only if there exists a sequence of Riemannian metrics $\left\{g_{j}\right\}$ for which the sectional/Ricci/scalar curvature is uniformly bounded, but their volumes $\left\{\operatorname{Vol}\left(M, g_{j}\right)\right\}$ approach zero as $j \rightarrow \infty$. If the curvature of $\left\{g_{j}\right\}$ only has a lower bound, we say that $M$ collapses with curvature bounded from below.

Define

$$
\operatorname{MinVol}(M):=\inf _{g}\left\{\operatorname{Vol}(M, g):\left|K_{g}\right| \leq 1\right\}
$$


and

$$
\operatorname{Vol}_{K}(M):=\inf _{g}\left\{\operatorname{Vol}(M, g): K_{g} \geq-1\right\}
$$

where the sectional curvature of the Riemannian metric $g$ is denoted by $K_{g}$, and we assumed the normalization $\operatorname{Vol}(M, g)=1$. The invariant $\operatorname{Min} \operatorname{Vol}(M)$ is known as the minimal volume and was introduced by Gromov [G82.

For a real number $D>0$, the more restrictive invariant $\mathrm{D}-\operatorname{Min} \operatorname{Vol}(\mathrm{M})$ of a closed smooth Riemannian manifold $(M, g)$ can be defined as follows. Much like $\operatorname{MinVol}(M)$, it is required that the infimum of $\operatorname{Vol}(M, g)$ is to be taken over all metrics $g$ and additionally requiring that the diameter remains bounded from above by $D$. The minimal entropy $\mathrm{h}(M)$ is the infimum of the topological entropy of the geodesic flow of a smooth metric $g$ on $M$ such that $\operatorname{Vol}(M, g)=1$. The inequality

$$
[\mathrm{h}(M)]^{n} \leq(n-1)^{n} \operatorname{MinVol}(M)
$$

is known to hold [PP03, page 417].

Let us now recall the definition of the Yamabe invariant B87, S87. Let

$$
\gamma:=[g]=\left\{u g: M \stackrel{u}{\rightarrow} \mathbb{R}^{+}\right\}
$$

be a conformal class of Riemannian metrics on $(M, g)$. The Yamabe constant of $(M, \gamma)$ is

$$
\mathcal{Y}(M, \gamma):=\inf _{g \in \gamma} \frac{\int_{M} S c a l_{g} d \operatorname{Vol}_{g}}{(\operatorname{Vol}(M, g))^{\frac{n-2}{n}}} .
$$

The Yamabe invariant of $M$ is defined as

$$
\mathcal{Y}(M):=\sup _{\gamma} \mathcal{Y}(M, \gamma) .
$$

A profound utility of $\mathcal{F}$-structures is sampled in the following results.

Theorem 1. Cheeger-Gromov CG86. The minimal volume of a manifold $M$ that admits a polarized $\mathcal{F}$-structure vanishes, and $M$ collapses with bounded sectional curvature.

Theorem 2. Paternain-Petean $\mathrm{PP} 03$. If a manifold $M$ admits an $\mathcal{F}$-structure then $\mathrm{h}(M)=0=\operatorname{Vol}_{K}(M)$ and $M$ collapses with sectional curvature bounded from below.

Collapse with scalar curvature bounded from below and collapse with bounded scalar curvature are equivalent for manifolds of dimension at least three [PP03, Proposition 7.1]. The latter is equivalent to the Yamabe invariant being nonnegative. The Yamabe invariant of a smooth closed manifold $M$ is positive if and only if $M$ admits a Riemannian metric of positive scalar curvature [B87. In particular, we have the following well-known lemma [B87, L99, PP03].

Lemma 1. Suppose $M$ is a closed smooth manifold that does not admit a Riemannian metric of positive scalar curvature. If $M$ collapses with bounded scalar curvature, then

$$
\mathcal{Y}(M)=0 .
$$

Moreover, any scalar-flat Riemannian metric on $M$ is Ricci-flat. 
2.3. Non-realization of Yamabe invariant zero in our examples. A compact orientable Riemannian n-manifold $M$ is $\epsilon^{-1}$-hyperspherical if there is a map onto the unit n-sphere that multiplies all distances by a factor less than or equal to $\epsilon>0$. If for every $\epsilon>0$, there exists a finite covering of $X$ that is $\epsilon^{-1}$-hyperspherical and spin, then $X$ is enlargeable GL80, Section 0]. Compact Euclidean space forms are examples of enlargeable manifolds, and enlargeability is a homotopy invariant. The non-realization of the Yamabe invariant for our examples in Theorem $\mathrm{A}$ and Corollary $\mathrm{B}$ is a corollary of the following result (cf. Lemma 1).

Theorem 3. Bieberbach, Gromov-Lawson [GL80] (cf. Schoen-Yau [SY79]). There are no Riemannian metrics of positive scalar curvature on a homotopy Euclidean space form M. Any Riemannian metric of non-negative scalar curvature on a homotopy Euclidean space form is flat. In such a case, $M$ is diffeomorphic to $\mathbb{E}^{n} / \Gamma$.

We finish this brief section with a pair of sample results.

Theorem 4. Hitchin [H74, Petean [P00]. Let $\Sigma$ be a homotopy n-sphere such that $\alpha(\Sigma) \neq 0$. Its Yamabe invariant is

$$
\mathcal{Y}(\Sigma)=0
$$

and it is not realized, i.e., there are no scalar-flat Riemannian metrics on $\Sigma$.

Proof. Petean has shown that the Yamabe invariant of a closed simply connected n-manifold with $n \geq 5$ is nonnegative [P00]. Hitchin has shown that $\alpha(\Sigma) \neq 0$ implies that $\Sigma$ does not admit a Riemannian metric of positive scalar curvature H74. Since the Yamabe invariant of a closed manifold is positive if and only if the manifold admits a Riemannian metric of positive scalar curvature, these results imply the vanishing $\mathcal{Y}(\Sigma)=0$. To argue that the Yamabe invariant is unrealized, we proceed by contradiction. Suppose there exists a scalar-flat Riemannian metric $g$ on $\Sigma$. Since $\alpha(\Sigma) \neq 0$ by assumption, the homotopy sphere has a non-trivial parallel spinor, and the Riemannian manifold $(\Sigma, g)$ thus has special holonomy [H74]. This is a contradiction, since homotopy spheres have generic holonomy. Hence, $\Sigma$ does not admit a scalar-flat Riemannian metric, and its Yamabe invariant is not realized.

It is unknown if the minimal entropy depends on the choice of smooth structure. In this paper, we provide a myriad of examples in terms of homeomorphism classes where it does not.

Proposition 4. There exists a smoothing $M$ of $\mathbb{C P}^{5}$ such that

$$
\mathrm{h}(M)=0=\operatorname{Vol}_{K}(M),
$$

and $M$ collapses with sectional curvature bounded from below.

Its Yamabe invariant is

$$
\mathcal{Y}(M)=0,
$$

and it is not realized, i.e., there are no scalar-flat Riemannian metrics on $M$.

Proof. Let $\Sigma$ be a homotopy 10-sphere such that $\alpha(\Sigma) \neq 0$; this sphere was proven to exist in M65, A66. The smooth manifold $M:=\mathbb{C P}^{5} \# \Sigma$ is homeomorphic to the complex projective 5-space. There is a nontrivial circle action on $\mathbb{C P}^{5}$ and on $\Sigma$ (see [572), and [PP03, Theorem 5.9] implies that there is a $\mathcal{T}$-structure on $M$. 
Theorems 2 implies that the minimal entropy of $M$ is zero, and that it collapses with sectional curvature bounded from below. In particular, $\mathcal{Y}(M) \geq 0$. This also follows from Petean's result [P00. Since $\alpha(M)=\alpha\left(\mathbb{C P}^{5} \# \Sigma\right)=\alpha\left(\mathbb{C P}^{5}\right)+\alpha(\Sigma)$ H74, it follows that $\mathcal{Y}(M)=0$. The claim about the nonexistence of scalar-flat Riemannian metrics on $M$ follows from the argument given in the proof of Theorem 4 (cf. Lemma 1).

2.4. Homotopy spheres and homotopy real projective spaces. Existence questions of circle actions on homotopy spheres has been a classical area of research since the late 1960's see MY68, S72] and references therein. These results yield We now mention some more constructions of $\mathcal{T}$-structures on closed smooth manifolds on the homeomorphism class of spheres and on the homotopy equivalence class of real projective spaces. A $\mathcal{T}$-structure can be constructed on any homotopy odddimensional sphere that bounds a parallelizable manifold as follows. The group of homotopy spheres that bound parallelizable manifolds are generated by the Kervaire spheres $\Sigma^{2 n-1}(d)$ KM63. All Kervaire spheres arise as Brieskorn manifolds in the following manner [B66, H66. Suppose $n \geq 3$ is an odd integer number, and $d= \pm 3$ mod 8. Then a homotopy sphere $\Sigma^{2 n-1}(d)$ arises as a real algebraic submanifold of $\mathbb{C}^{n+1}$ defined by the equations

$$
z_{0}^{d}+z_{1}^{2}+\ldots+z_{n}^{2}=0
$$

and

$$
\left|z_{0}\right|^{2}+\left|z_{1}\right|^{2}+\ldots+\left|z_{n}\right|^{2}=1 .
$$

Scalar multiplication on $\mathbb{C}^{n+1}$ yields a circle action on $\Sigma^{2 n-1}(d)$ and using a result of Paternain-Petean [PP03, Theorem 5.1]. we conclude that every homotopy odddimensional sphere that bounds a parallelizable manifold admits a $\mathcal{T}$-structure. A polarized $\mathcal{T}$-structure on these manifolds is constructed using pseudo-free circle actions as in Example2 Montgomery-Yang have shown that all homotopy 7-spheres admit such an action MY68.

The $\mathcal{T}$-structure on the Brieskorn manifolds descend to a $\mathcal{T}$-structure on closed manifolds that are homotopy equivalent to $\mathbb{R} P^{2 n-1}$. Indeed, there is an orientationpreserving fixed point involution $T: \Sigma^{2 n-1}(d) \rightarrow \Sigma^{2 n-1}(d)$ given by

$$
\left(z_{0}, z_{1}, z_{2}, \ldots, z_{n-1}, z_{n}\right) \mapsto\left(z_{0},-z_{1},-z_{2}, \ldots,-z_{n-1},-z_{n}\right) .
$$

The circle action commutes with the involution and yields a $\mathcal{T}$-structure on the orbit space $\Sigma^{2 n-1}(d) / T$. The latter is a closed manifold that is homotopy equivalent to $\mathbb{R} P^{2 n-1}$ yet it need not be homeomorphic nor diffeomorphic to it [LM71.

Another construction of $\mathcal{T}$-structures arises as a generalization of Example 1 in terms of a broader set of choices for the decomposition of $S^{n}$ into two building blocks that was given in (6), and the different choices of diffeomorphisms that are used to identify the pieces together. Consider now

$$
S^{n}=\partial D^{n+1}=\partial\left(D^{k} \times D^{n+1-k}\right)=\left(S^{k-1} \times D^{n+1-k}\right) \cup_{\text {id }}\left(D^{k} \times S^{n-k}\right) .
$$

Build a homotopy sphere

$$
\Sigma^{n}=\left(S^{k-1} \times D^{n+1-k}\right) \cup_{\varphi_{k, n}}\left(D^{k} \times S^{n-k}\right)
$$


such that the diffeomorphism of the common boundaries

$$
\varphi_{k, n}: S^{k-1} \times S^{n-k} \rightarrow S^{k-1} \times S^{n-k}
$$

satisfies $\pi_{S^{k-1}} \circ \varphi_{k, n}=\pi_{S^{k-1}}$, where $\pi_{S^{k-1}}$ is the projection to the sphere factor GW97. A $\mathcal{T}$-structure is given by taking the open cover $U_{1}:=S^{k-1} \times D^{n+1-k}$ and $U_{2}:=D^{k} \times S^{n-k}$ and choosing torus actions that commute with the gluing diffeomorphism. The Gromoll groups indicate when the homotopy n-sphere (46) is exotic [GW97, Section 3].

2.5. Homotopy Euclidean space forms. The main result of this section is Theorem 6. In order to share light on it, we begin by discussing homotopy tori. As it was mentioned in the introduction, Casson, Wall and Hsiang-Shaneson have shown existence of inequivalent smoothings (up to diffeomorphism) and inequivalent PLstructures (up to PL-homeomorphism) on homotopi tori. The fundamental theorem of smoothing theory states that a topological manifold of dimension at least five admits a smooth structure if and only if its topological tangent bundle admits the structure of a vector bundle. A homotopy n-torus with a PL-structure is parallelizable, hence smoothable W99, Chapter 15A]. The set $\mathcal{S}^{P L}\left(T^{n}\right)$ of equivalence classes up to PL-homeomorphism of closed n-dimensional PL-manifolds that are homotopy equivalent to the n-torus is in bijective correspondence with $H^{3}\left(T^{n} ; \mathbb{Z} / 2\right)$ W69, HS69, W99. There is an isomorphism

$$
\left[T^{n}, G / P L\right] \cong \bigoplus_{0 \leq i \leq n} H^{i}\left(T^{n} ; \pi_{i}(G / P L)\right),
$$

where the groups $\pi_{i}(G / P L)$ are finite. According to Wall W69, Corollary] W69, Page 236] and Hsiang-Shaneson [HS69, Theorem B], a closed n-dimensional PLmanifold that is homotopy equivalent to the n-torus with $n \geq 5$ is finitely covered by the standard $T^{n}$. The normal PL-invariants are natural for coverings and any element of the group $H^{r}\left(T^{n} ; A\right)$ with $A$ finite is killed by passing to a finite cover (an argument to show this will be given in the proof of Theorem 6). Coverings corresponding to subgroups of $\pi_{1}=\mathbb{Z}^{n}$ of odd index are fake tori; those that correspond to subgroups of even index are standard. The set of smoothings on a PL-structure class are in bijective correspondence with elements of

$$
\left[T^{n}, P L / O\right] \cong \bigoplus_{i \leq n} H^{i}\left(T^{n} ; \pi_{i}(P L / O)\right)
$$

and the normal invariants, as in the PL-structures case, are natural for coverings W99]; indeed, the homotopy groups of the loop space $P L / O$ are finite and any element of the cohomology group of a homotopy n-torus with coefficients in the finite group $\pi_{i}(P L / O)$ can be killed by passing to a finite cover. We are indebted to Terry Wall for explaining this to us. Jim Davis [FKSD13, Appendix] has an excellent synthesized description of these properties, and his synthesis will be used in the proof of Theorem [6. Corollary $\mathrm{B}$ and Proposition $\mathrm{C}$ are proven using the following result.

Theorem 5. Hsiang-Shaneson [HS69], Wall W69, W99]. For $n \geq 5$, every homotopy $n$-torus is finitely covered by the standard $T^{n}$.

Let us now move into homotopy Euclidean space forms in full generality. Regarding the structure of their topological tangent bundle, the reader is directed to [65] and we now consider smoothable homotopy Euclidean space forms. We now 
present the main result in this section, which is a consequence of the aforementioned results of Hsiang-Shaneson and Wall.

Theorem 6. Let $M$ be a homotopy Euclidean space form $\mathbb{E}^{n} / \Gamma$. There is a homeomorphism

$$
\varphi: M \rightarrow \mathbb{E}^{n} / \Gamma
$$

and a finite cover

$$
T^{n} \rightarrow \mathbb{E}^{n} / \Gamma
$$

so that the induced pullback homeomorphism

$$
\widetilde{M} \rightarrow T^{n}
$$

is isotopic to a diffeomorphism.

The existence of the homeomorphism (50) was established by Farrell-Jones [FJ98, where they showed that any homotopy equivalence between such manifolds is isotopic to a homeomorphism. One of the Bieberbach Theorems states the existence of the finite covering (51). It is immediate to see that Theorem 6 follows from Theorem 5 . The justification for including the following proof is to flesh out details of the results in surgery theory that are being employed. We built greatly on work of Jim Davis [FKSD13, Appendix] for the following description.

Proof. We use work of Jim Davis to prove that the pullback homeomorphism (52) is isotopic to a diffeomorphism. Two homeomorphisms are isotopic if there exists a homotopy between them that consists of homeomorphisms. We commence by recalling a necessary property.

Condition 1. For any $i>0$, for any finite abelian group $A$, for any finite cover $\hat{p}: \hat{N} \rightarrow N$, and for any element $x \in H^{i}(\hat{N} ; A)$, there exists a finite cover $\tilde{p}: \widetilde{N} \rightarrow$ $\hat{N}$ such that $\tilde{p}^{*} x=0$.

An extension of the described at the beginning of the section for homotopy tori is the following lemma, which is the main ingredient to prove Theorem 6

Lemma 2. Davis [FKSD13, Lemma A.3]. Let $\varphi: M \rightarrow N$ be a homeomorphism of closed smooth $n$-manifolds with $n \geq 5$, and assume $N$ satisfies Condition 1 . There exists a finite cover

$$
\hat{N} \rightarrow N
$$

such that the induced pull-back homeomorphism

$$
\hat{M} \rightarrow \hat{N}
$$

is isotopic to a diffeomorphism.

To prove Theorem 6, we need to show that a homotopy Euclidean form satisfies Condition [1 in order to invoke Lemma 2.

Claim 1. Any Euclidean space form $\mathbb{E}^{n} / \Gamma$ satisfies Condition 1 .

One of the Bieberbach theorems states that there is a finite covering of $\mathbb{E}^{n} / \Gamma$ that is isometric to the n-torus. Thus, in order to prove Claim 1, it suffices to check that $T^{n}$ satisfies the following condition. 
Condition 2. For any $i>0$, for any finite abelian group $A$, and for any element $x \in H^{i}(N ; A)$, there exists a finite cover $\hat{p}: \hat{N} \rightarrow N$ such that $\hat{p}^{*} x=0$.

We proceed to show that the n-torus satisfies Condition 2 with $\hat{N}=T^{n}=N$. The following argument fills in the details of the description of the normal invariants of a homotopy n-torus given at the beginning of the section. For $i=1$, we have the commutative diagram

$$
\begin{array}{ccccc}
H^{1}(T ; A) & \cong & \operatorname{Hom}\left(\pi_{1}\left(T^{n}\right), A\right)= & \operatorname{Hom}\left(\mathbb{Z}^{n}, A\right) \\
\downarrow \hat{p}^{*} & & & \downarrow-\circ \hat{p}_{*} & \\
H^{1}\left(T^{n} ; A\right) & \stackrel{\cong}{\longrightarrow} & \operatorname{Hom}\left(\pi_{1}\left(T^{n}\right), A\right)= & \operatorname{Hom}\left(\mathbb{Z}^{n}, A\right)
\end{array}
$$

that show $\hat{p}^{*} x=0$ for every element $x \in H^{1}\left(T^{n}, A\right)$.

We now argue in the case $i \geq 2$ by induction on $n$. A free circle action on $T^{n}$ gives rise to a principal $S^{1}$-bundle $S^{1} \rightarrow T^{n} \rightarrow T^{n} / S^{1}=T^{n-1}$. Suppose that Condition 2 holds for any torus of smaller dimension. Canonically associated to the principal $S^{1}$-fibration we have DK01

$$
\cdots \longrightarrow H^{i-2}\left(T^{n-1} ; A\right) \stackrel{\cup e}{\longrightarrow} H^{1}\left(T^{n-2} ; A\right) \stackrel{\pi^{*}}{\longrightarrow} H^{i}\left(T^{n} ; A\right) \stackrel{\pi_{!}}{\longrightarrow} H^{i-1}\left(T^{n-1} ; A\right) \longrightarrow \cdots
$$

the exact Gysin sequence. Our hypothesis on the dimension implies the existence of a cover $\tilde{p}: T^{n-1} \rightarrow T^{n-1}$ so that $\tilde{p}^{*}(\pi ! x)=0$ for any element $H^{i}\left(T^{n-1} ; A\right)$ with $i \geq 2$. Consider now the diagram

$$
\begin{aligned}
& T^{n} \stackrel{\cong}{\longrightarrow} T^{n} / S^{1}=T^{n-1} \\
& \downarrow \tilde{p} \quad \downarrow \tilde{p}_{S^{1}} \\
& T^{n} \stackrel{\cong}{\longrightarrow} T^{n} / S^{1}=T^{n-1} \text {. }
\end{aligned}
$$

It yields a map of principal $S^{1}$-bundles and a map of exact Gysin sequences. It is now immediate to conclude that $T^{n}$ satisfies Condition 2, thus $\mathbb{E}^{n} / \Gamma$ satisfies Condition 1, and Lemma 2 is ready to be invoked. This concludes the proof of Theorem 6 .

We now proceed to prove that any smooth manifold homeomorphic to a highdimensional torus can be equipped with a polarized $\mathcal{F}$-structure.

Corollary 2. Every homotopy Euclidean $n$-space form with $n \geq 5$ admits a polarized $\mathcal{F}$-structure.

In particular, every smoothing and every PL-structure on the homeomorphism type of $T^{n}$ with $n \geq 5$ admits a polarized $\mathcal{F}$-structure.

Proof. The proposition follows from Proposition 3 and Theorem 6 .

Examples of inequivalent smoothings on homotopy Euclidean space forms were described in Corollary 1. We finish the section by mentioning an explicit construction of inequivalent PL-structures on homotopy Euclidean space forms.

Example 3. Fake tori. The connected sum construction (29) with $X=T^{n}$ of homotopy n-tori that was described in Corollary 1 produces inequivalent smoothings but not inequivalent PL-structures. For any homotopy sphere $\Sigma^{n}$, the manifolds $T^{n} \# \Sigma^{n}$ and $T^{n}$ are PL-homeomorphic by the Alexander trick. Fake n-tori are constructed as mapping tori

$$
M_{h}:=[0,1] \times T^{n} /(1, x)(0, h(x))
$$


for a diffeomorphism $h: T^{n} \rightarrow T^{n}$, as described in Farrell-Gogolev [FG14. If the diffeomorphism $h$ is not PL pseudo-isotopic to the identity map $i d_{T^{n}}$, then the mapping torus $M_{h}$ is a fake torus [FG14, Corollary 2.3]. The fake torus $M_{h}$ is the total space of a $T^{n}$-bundle over the circle. There is a bijection between the smooth pseudo-isotopy classes of diffeomorphisms $h$ whose induced homomorphisms on fundamental groups satisfy $h_{*}=i d_{\pi_{1}\left(T^{n}\right)}$, and the smooth structures $\theta$ on a homotopy torus $M$ such that the inclusion map $\sigma: T^{n} \times\{0\} \hookrightarrow\left(M \times S^{1}, \theta\right)$ is a smooth embedding. The smooth structures correspond to the subgroup of elements $\varphi \in\left[T^{n+1}, T O P / O\right]$ such that $\varphi \circ \sigma$ is null-homotopic W99, FG14. It is straight-forward to construct a polarized $\mathcal{T}$-structure on $M_{h}$ using the constructions presented in Section 2.1

\section{Proofs of Results Stated in the introduction}

3.1. Proof of Theorem $\mathbf{A}$ and Corollary B, Homotopy Euclidean space nforms admit a polarized $\mathcal{F}$-structure by Proposition 3 and Theorem 6 as stated in Corollary 2, Their minimal volume is zero and they collapse with bounded sectional curvature by Theorem 1 which implies that their Yamabe invariant is nonnegative as indicated in Lemma 1. Such a manifold does not admit a Riemannian metric of positive scalar curvature [GL80, as stated in Theorem 3. Hence, we conclude that a homotopy Euclidean space form has zero Yamabe invariant. Other than the standard $\mathbb{E}^{n} / \Gamma$, no homotopy Euclidean space form admits a scalar-flat Riemannian metric by Theorem 3 .

Remark 1. Jim Davis has shown in [FKSD13, Appendix] that a smooth manifold of dimension at least five and that is homeomorphic to a nilmanifold has a finite covering which is diffeomorphic to a nilmanifold. The existence of a polarized $\mathcal{F}$ structure on such a manifold can be proven using tweaks to the arguments and constructions that were presented in Section 2.1. The Yamabe invariant on this homeomorphism class is zero and it is not realized by any smoothing since a smooth manifold that is homeomorphic to a nilmanifold does not admit Ricci-flat metrics.

3.2. Proof of Proposition $\mathbf{C}$. This result is a produce of the work of BauesTuschmann BT12, Theorem A, and Theorem 5 . Indeed, let $M$ be an exotic/fake torus that is not a product of standard and a lower-dimensional exotic/fake tori; exotic tori of this kind are considered in Corollary 10 and Example 3 BauesTuschmann have shown that

$$
\operatorname{D-Min} \operatorname{Vol}(M)>0
$$

On the other hand,

$$
\mathrm{D}-\operatorname{Min} \operatorname{Vol}\left(T^{n}\right)=0 \text {. }
$$

Since any homotopy n-tori is finitely covered by the standard n-torus, the proposition follows.

Remark 2. Baues-Tuschmann asked in [BT12, Question 1.7] if an exotic/fake torus that is not a product of a standard torus and a lower-dimensional exotic/fake torus always has non-vanishing minimal volume. Corollary $B$ answers their question in the negative. As we have mentioned in the introduction, they have shown that the finer invariant D-MinVol does discern the standard smooth structure within a subset of homotopy tori. Corollary $B$ states that it is the realization of the Yamabe invariant that tells the standard smooth structure apart from any other. 
3.3. Proof of Theorem D. A polarized $\mathcal{T}$-structure was constructed on $M(G)$ in Proposition 1 The claims concerning its minimal volume and collapse follow from Theorem 1

\section{AcKnowledgements}

We are indebted to Jim Davis for kindly bringing [FKSD13, Appendix] to our attention, without which we could have not proven our main result as stated. We are grateful to an anonymous referee for kindly pointing out a mistake on a previous version of the manuscript. We thank Frank Quinn, Andrew Ranicki, and Terry Wall for helpful e-mail correspondence. We thank Bernd Ammann, Oliver Baues, Fernando Galaz-García, Marco Radeschi, and Wilderlich Tuschmann for interesting conversations. We gratefully acknowledge support from the University of Fribourg and the organizers of its Riemannian Topology Seminar 2015 for a very pleasant and productive meeting during which part of this paper was written.

\section{REFERENCES}

[A66] J. F. Adams, On the groups $J(X)$. IV, Topology 5 (1966), 21 - 71.

[ABK70] P. L. Antonelli, D. Burghelea, and P. J. Kahn, Gromoll groups, Diff $S^{n}$ and bilinear constructions of exotic spheres, Bull. Amer. Math. Soc. 76 (1970), 772 - 777.

[BT12] O. Baues and W. Tuschmann, Seifert fiberings and collapsing infrasolv spaces, arXiv:1209.2450v1 (2012).

[B87] A. L. Besse, Einstein manifolds, Ergebnisse der Mathematik und ihrer Grenzgebiete Springer-Verlag, Berlin, 1987. xii +510 .

[B98] A. L. Bessières, Un théorème de rigidité différentielle, Comment. Math. Helv. 73 (1998), $443-479$.

[B66] E. Brieskorn, Beispiele zur differentialtopologie von singularitäten, Invent. Math 2 (1996), $1-14$.

[CG86] J. Cheeger and M. Gromov, Collapsing Riemannian manifolds while keeping their curvature bounded I, J. Diff. Geom. 23 (1986), 309 - 346.

[CR96] J. Cheeger and X. Rong, Existence of polarized F-structures on collapsed manifolds with bounded curvature and diameter, Geom. Func. Anal. 6 (1996), 411 - 429.

[DK01] J. F. Davis and P. Kirk, Lecture notes in algebraic topology, Graduate Studies in Mathematics 35, Amer. Math. Soc. Providence, RI, 2001. xvi+367 pp.

[D12] M. Dehn, Über unendliche diskontinuierliche Gruppen, Math. Annalen 71 (1912), 16 - 144.

[FG14] F. T. Farrell and A. Gogolev, Examples of expanding endomorphisms on fake tori, J. Topol. 7 (2014), 805 - 816.

[FJ98] F. T. Farrell and L. E. Jones, Rigidity for aspherical manifolds with $\pi_{1} \subset G L_{m}(\mathbb{R})$, Asian J. Math. 2 (1998), 215 - 262.

[FKSD13] D. Fischer, B. Kalinin, and R. Spatzier with an appendix by J. F. Davis, Global Rigidity of higher rank Anosov actions on tori an nilmanifolds, J. Amer. Math. Soc. 26 (2013), 167 198.

[GS99] R. E. Gompf and A. I. Stipsicz, 4-manifolds and Kirby calculus, Graduate Studies in Mathematics 20, Amer. Math. Soc. Providence, RI, 1999. xvi+558 pp.

[G82] M. Gromov, Volume and bounded cohomology, Publ. Math. Inst. Hautes Etud. Sci. 56 (1982), 1- 99.

[GL80] M. Gromov and H. B. Lawson, Spin and scalar curvature in the presence of a fundamental group. I, Ann. of Math. Vol. 111 (1980), 209 - 230.

[GW97] K. Grove and F. Wilhelm, Metric constraints on exotic spheres via Alexandrov geometry, J. Reine Angew. Math. 487 (1997), 201 - 217.

[H66] F. Hirzebruch, Singularities and exotic spheres, Séminaire Bourbaki 19e année, 1966/67, No. 314 , p. 13 - 32 .

[H74] N. Hitchin, Harmonic spinos, Advances in Math. 14 (1974), 1 - 55.

[HS69] W. C. Hsiang and J. L. Shaneson, Fake tori, the Annulus conjecture, and the Conjectures of Kirby, Proc. Nat. Acad. Sci. U. S. A Vol. 62 (1969), 687 - 691. 
[HW69] W. C. Hsiang and C. T. C. Wall, On homotopy tori II, Bull. London Math. Soc. 1 (1969), $341-342$.

[KM63] M. A. Kervaire and J. Milnor, Groups of homotopy spheres I Ann. of Math. 77 (1963), $504-537$.

[L99] C. LeBrun, Kodaira dimension and the Yamabe problem, Comm. Anal. Geom. 7 (1999), $1119-1125$.

[LM71] S. López de Medrano, Involutions on manifolds, Ergebnisse der Mathematik und ihrer Grenzgebiete 59, Springer-Verlag, New York-Heidelberg, 1971. ix + 103 pp.

[M56] J. Milnor, On manifolds homeomorphic to the 7-sphere, Ann. of Math. 64 (1956), 399 405.

[M65] J. Milnor, Remarks concerning spin manifolds, Differential and Combinatorial Topology, 55 - 62, Princeton Univ. Press, Princeton, NJ, 1968.

[MY68] D. Montgomery and C. T. Yang, Differentiable actions on homotopy seven spheres II, Proc. Conf. on Transformation Groups (1967), 125 - 134. Springer-Verlag, New York, 1968.

[PP03] G. P. Paternain and J. Petean, Minimal entropy and collapsing with curvature bounded from below, Invent. Math. 151 (2003), 415 - 450.

[PP09] G. P. Paternain and J. Petean, Collapsing manifolds obtained by Kummer-type constructions, Trans. Amer. Math. Soc. 361 No. 8 (2009), 4077 - 4090.

[P00] J. Petean, The Yamabe invariant of simply connected manifolds, J. Reine Angew. Math. 523 (2000), $225-231$.

[S87] R. Schoen, Variational theory for the total scalar functional for Riemannian metrics and related topics, Springer Lect. Notes Math. 1365 (1987), 120 - 154.

[SY79] R. Schoen and S. T. Yau, On the structure of manifolds with positive scalar curvature, Manuscripta Math. 28 (1979), 159 - 183.

[S72] R. Schultz, Circle actions on homotopy spheres bounding plumbing manifolds, 36 (1972), $297-300$.

[S81] T. Soma, The Gromov invariant of links, Invent. Math. 64 (1981), 445 - 454.

[T65] J. A. Thorpe, Parallelizability and flat manifolds, Proc. Amer. Math. Soc. 16 (1965), 138 142.

[W69] C. T. C. Wall, On homotopy tori and the annulus theorem, Bull. London Math. Soc. 1 (1969), 95 - 97.

[W99] C. T. C. Wall, Surgery on compact manifolds Second Ed. Edited and with a foreword by A. A. Ranicki. Mathematical Surveys and Monographs, 69. Amer. Math. Soc., Providence, RI, 1999. xvi + 203 pp.

Scuola Internazionale Superiori di Studi Avanzati (SISSA), Via Bonomea 265, 34136, Trieste, Italy

E-mail address: rtorres@sissa.it 